\title{
A comparative study between a single dose pre-induction antibiotic versus continuous antibiotics to prevent urinary tract infection during ureteroscopy
}

\author{
Rasti Karim Mama ${ }^{1 *}$, Pishtewan Hashim Al-bazzaz ${ }^{2}$ and Nihad Pauls Al-Ibraheem ${ }^{2}$ \\ ${ }^{1}$ Departement of Urology, Rizgary Teaching Hospital, Erbil, Iraq \\ ${ }^{2}$ Department of Urology, College of Medicine, Hawler Medical University, Erbil, Iraq
}

\begin{abstract}
Background and objective: This study aimed to evaluate if the addition of postoperative antibiotics reduces the incidence of symptomatic urinary tract infections following ureteroscopic stone treatment beyond the recommended preoperative dose.

Methods: This prospective comparative study was conducted between March and November 2017. A total of 80 patients with preoperative negative urine culture undergoing ureteroscopy were randomly allocated. All the patients received single dose antibiotic before the induction of anesthesia and a group of them also received postoperative antibiotics. The rate of symptomatic urinary tract infection was compared between the patients who received only preoperative antibiotics (group 1 ) and those who received pre- and postoperative antibiotics (group 2).

Results: Eighty patients underwent ureteroscopy for stone treatment, the time to follow up was $4 \pm 2$ weeks from ureteroscopy. Eleven (13.8\%) patients in total developed urinary tract infections postoperatively, including five (12.5\%) from group 1 and six (15\%) from group 2, $\mathrm{P}=0.74$. In group 1, four patients presented with cystitis and one with sepsis; those patients with infections in group 2 presented with pyelonephritis $(n=2)$ and cystitis $(n=4)$. Risk factors such as preoperative stenting, nephrostomy tubes, and Foleys catheters neither differed between groups nor did they predispose patients to postoperative infections.
\end{abstract}

Conclusions: The postoperative symptomatic urinary tract infection rate in this study (13.8\%) is in agreement with previous reports. This study suggests that a single preoperative dose of antibiotics is sufficient, and additional postoperative antibiotics do not decrease infection rates after ureteroscopic stone treatment.

\section{Introduction}

Ureteroscopic lithotripsy is a common urological treatment for ureteric stones. This procedure poses a risk of postoperative urinary tract infection (UTI) of up to $25 \%$ in patients without prophylactic antibiotics [1,2]. This is primarily attributed to the introduction of bacteria into the urinary tract upon insertion of surgical instruments. Any intravasation of bacteria or endotoxins into the blood stream may lead to urosepsis, a potentially lethal complication. These infections carry significant morbidity and increased healthcare expenditures $[3,4]$.

Stone intervention should not be underestimated. All patients should be evaluated with a complete medical history, proper physical examination, and laboratory tests, including midstream urine (MSU) culture and sensitivity. A full preoperative evaluation will identify high-risk patients with the potential for infectious complications including Immunosuppression, Diabetes mellitus, female gender, anatomic anomalies, voiding dysfunction, urinary diversion, urinary tract obstruction and presence of indwelling tubes (stent, catheter, nephrostomy) [5-7].

To decrease the risk for infection following ureteroscopy, optimizing modifiable risk factors is ideal. Furthermore, antimicrobial prophylaxis is recommended by the American Urology Association (AUA) Best Practice Guidelines to decrease further the risk of postoperative infection in patients undergoing ureteroscopy. Preoperative prophylactic antibiotics play an important role in reducing postoperative infections.
AUA guidelines recommend a single dose use of antibiotic covering both positive and negative uropathogens on all patients taking ureteroscopic lithotripsy [8].

This study aimed to evaluate the effect of adding postoperative antibiotics on reducing urinary tract infections (UTIs) following ureteroscopic stone treatment beyond the recommended preoperative dose.

\section{Material and methods}

This prospective comparative study was conducted in Rizgary Teaching Hospital, Erbil, Kurdistan region-Iraqfrom March to November 2017. A total of 80 patients with ureteric stones and sterile urine were scheduled to receive ureteroscopic lithotripsy in. All the patients were received a single dose of i.v gentamycin $(80 \mathrm{mg})$, ceftriaxone $(1 \mathrm{~g})$ or Ciprofloxacin $(400 \mathrm{mg})$ before starting the induction of anesthesia. A group of patients received only preoperative antibiotics (group 1), while in addition, a subset of patients also received postoperative antibiotics (gentamycin, ciprofloxacin or cefixime) (group 2). The preoperative

${ }^{*}$ Correspondence to: Rasti Karim Mama, Department of urology, Rizgary Teaching Hospital, Erbil, Iraq, Tel: 009647504453406; E-mail: Rasty_Karim@yahoo.com

Key words: antibiotics, infection, prophylaxis, stones, ureteroscopy

Received: September 12, 2018; Accepted: September 24, 2018; Published: September 26, 2018 
Mama RK (2018) A comparative study between a single dose pre-induction antibiotic versus continuous antibiotics to prevent urinary tract infection during ureteroscopy

patient evaluation included a thorough history taking, physical examinations, blood chemistry tests, urine analysis and urine culture. Exclusion criteria were: age $<18$, preoperative positive urine cultures and abnormal renal function. All the procedures were done under general anesthesia by using semirigid ureteroscope 9.5Fr., pneumatic Lithoclast was used as intracorporial lithotripter and Dormia basket was used for extraction of stone fragments. A DJ stent was inserted at the end of the procedure according to the surgeon's discretion.

Our primary end point was symptomatic UTI. If any symptoms of UTI developed (pain, urgency, frequency, dysuria, fever) during follow up, a urine culture was repeated.

A specially designed questionnaire was used for collectiing data. The data were entered in the computer and then analyzed by the statistical package for the social sciences (version 22). The results were compared between patients with different variables, with a statistical significance level of $<0.05$.

The results were presented as rates, ratios, frequencies, percentages in tables and figures and analyzed using the t-test and Chi square test.

The study was approved by the scientific and the ethics committees at the Kurdistan Board for Medical Specialties.

\section{Results}

Over the period of the study, 80 patients underwent ureteroscopic lithotripsy at our institution, the median age was 41.8 (range: 23-74), and male to female ratio was 43:37. Mean BMI was comparable between the two groups; group 1 had a BMI of 25.83 (standard deviation $[\mathrm{SD}]=4.107)$ and group $2 \mathrm{had}$ a $\mathrm{BMI}$ of $27.75(\mathrm{SD}=5.113)$. Both groups were similar in terms of basic demographics (Table 1).

The preoperative antibiotic selection was predominantly gentamycin in $47(58.8 \%)$ patients followed by ciprofloxacin in $17(21.3 \%)$ patients and ceftriaxone in $16(20 \%)$ patients (Figure 1). Ciprofloxacin was most commonly used in $57 \%$ patients followed by gentamycin in $22.50 \%$ patients and cefixime in $20 \%$.

Overall, eleven patients had a clinical UTI postoperatively or positive urine culture (Figure 2).

Five $(12.5 \%)$ of these patients were in group 1 . One of them presented with urosepsis and the other four with cystitis. The uroseptic patient was culture positive for Klebsiella while E. coli was positive in three patients and Pseudomonas aeruginosa in one patient with cystitis.

Six (15\%) patients in group 2 clinically demonstrated signs of a UTI; two patients presented with pyelonephritis, one of them urine culture was positive for E. coli, the other was urine culture negative. The other four patients presented with cystitis; two of them urine culture was positive for $E$. coli, urine culture for one of the other two patients was positive for Enterococcus and the other one was negative culture. All were treated effectively.

Table 1. Demographic characteristics of the participants

\begin{tabular}{|c|c|c|c|c|c|}
\hline Variable & Groups & $\mathbf{N}$ & Mean/N & S.D/\% & P value \\
\hline \multirow{2}{*}{ Age } & Group 1 & 40 & 41.08 & 12.855 & \\
\cline { 2 - 5 } & Group 2 & 40 & 41.88 & 11.791 & 0.77 \\
\hline \multirow{2}{*}{ BMI } & Group 1 & 40 & 25.83 & 4.107 & \multirow{2}{*}{0.06} \\
\cline { 2 - 5 } & Group 2 & 40 & 27.75 & 5.113 & \\
\hline \multirow{3}{*}{ Gender } & \multirow{3}{*}{ Group 1 } & Male & 21 & $52.5 \%$ & \multirow{2}{*}{0.82} \\
\cline { 2 - 5 } & & Female & 19 & $47.5 \%$ & \\
\cline { 2 - 5 } & \multirow{2}{*}{ Group 2 } & Male & 22 & $55 \%$ & \multirow{2}{*}{} \\
\cline { 3 - 6 } & & Female & 18 & $45 \%$ & \\
\hline
\end{tabular}

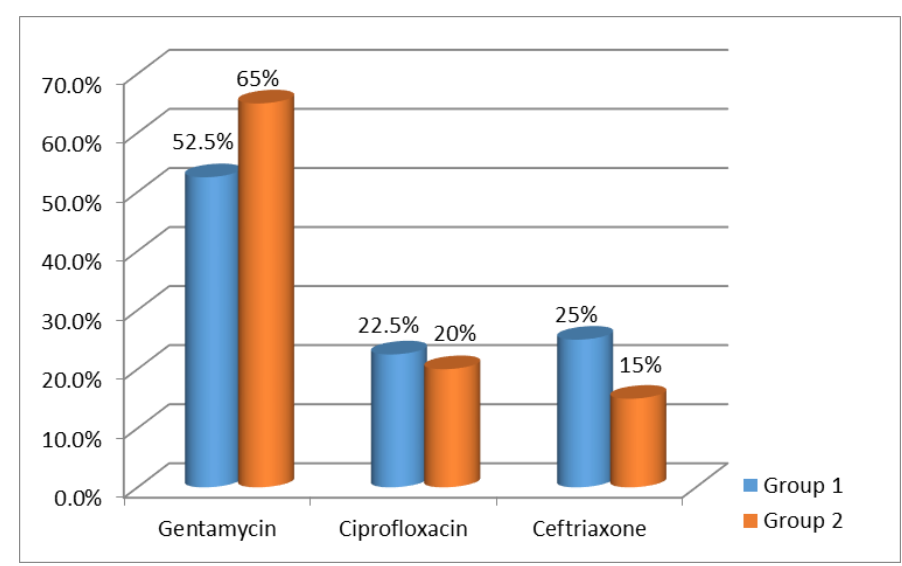

Figure 1. Preoperative antibiotic use for Group 1 and Group 2 patients

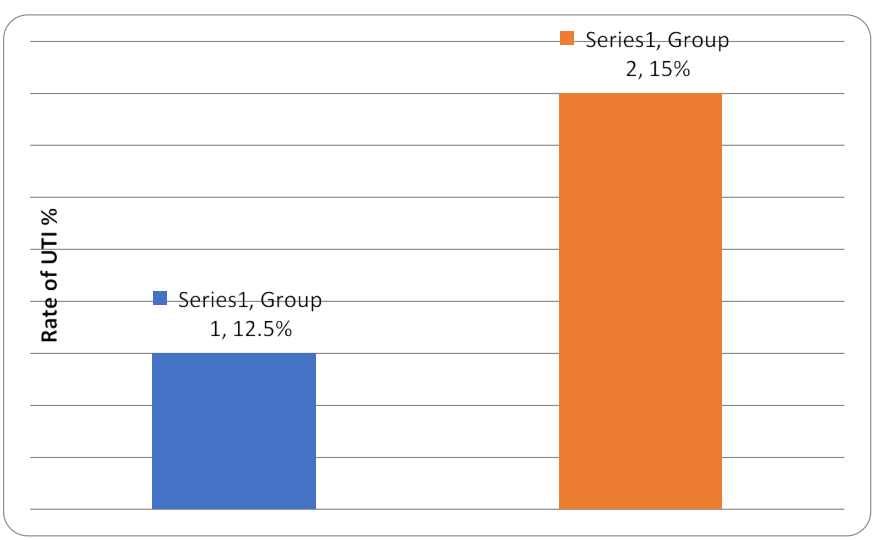

Figure 2. Post-operative UTI of both Groups ( $\mathrm{P}=074)$

Surgical factors and risk factors for infections were also assessed, as demonstrated in (Table 2). No associations with infection could be made here.

\section{Discussion}

Retrograde ureteroscopy as a method of treatment for ureteral calculi can lead to infectious complications. In our study 11(13.8\%) patients were treated for cystitis, pyelonephritis, or urosepsis since their ureteroscopy was performed. This compares with the historical rate of UTI after ureteroscopic management for the stone disease for the distal, middle, and upper ureter is $4 \%, 2 \%$, and $4 \%$, respectively [911]. Among studies with less stringent exclusion criteria or inclusion of stenting, the rate of UTI or fever is comparable at $9 \%$ to $10 \%$, and $33 \%$, respectively $[12,13]$. This elevated rate of infection may be attributable to the inclusion of patients with risk factors for postoperative infections described in Table 2.

Among preoperatively stented patients, three of twelve (25\%) presented with postoperative infection. Mechanistically, this could occur as a result of a biofilm being present on the stent, as being reported to occur in up to $25 \%$ of cases depending on indwelling duration; however, positive urine cultures are only found among $10 \%$ of patients with stent colonization $[14,15]$. Several studies looking at the colonization of ureteral stents upon removal have shown that a significant number of stents are colonized despite the use of prophylactic antibiotics, suggesting that prophylaxis is ineffective to prevent the interaction of bacteria with the surfaces of stents [16-18]. The fact that, however, in these studies and routine practice, the presence of a bacterial biofilm 

ureteroscopy

Table 2. Surgical factors and infection risk factors compared among patients receiving only preoperative antibiotics (Group 1) to those receiving Pre- and postoperative antibiotics (Group 2)

\begin{tabular}{|c|c|c|c|c|c|c|}
\hline \multirow{3}{*}{ Variable } & \multirow{3}{*}{ Categories } & \multicolumn{4}{|c|}{ Study samples } & \multirow{3}{*}{ P-value } \\
\hline & & \multicolumn{2}{|c|}{ Group 1} & \multicolumn{2}{|c|}{ Group 2} & \\
\hline & & No. & $(\%)$ & No. & $(\%)$ & \\
\hline \multirow{2}{*}{ Postoperative Foley's catheter } & Yes & 1 & $(2.5)$ & 2 & $(5.0)$ & \multirow{2}{*}{0.55} \\
\hline & No & 39 & $(97.5)$ & 38 & $(95.0)$ & \\
\hline \multirow{2}{*}{ Pre-operative stent } & Yes & 8 & $(20.0)$ & 4 & $(10.0)$ & \multirow{2}{*}{0.21} \\
\hline & No & 32 & $(80.0)$ & 36 & $(90.0)$ & \\
\hline \multirow{2}{*}{ Postoperative stent } & Yes & 32 & $(80.0)$ & 34 & $(85.0)$ & \multirow{2}{*}{0.55} \\
\hline & No & 8 & $(20.0)$ & 6 & $(15.0)$ & \\
\hline \multirow{2}{*}{ Diabetes mellitus } & Yes & 1 & $(2.5)$ & 0 & $(0.0)$ & \multirow{2}{*}{0.31} \\
\hline & No & 39 & $(97.5)$ & 40 & $(100.0)$ & \\
\hline \multirow{2}{*}{ Ureteroscopy side } & Right & 19 & $(47.5)$ & 21 & $(52.5)$ & \multirow{2}{*}{0.65} \\
\hline & Left & 21 & $(52.5)$ & 19 & $(47.5)$ & \\
\hline \multirow{3}{*}{ Stone location } & Upper ureter & 8 & $(20.0)$ & 12 & $(30.0)$ & \multirow{3}{*}{0.17} \\
\hline & Mid ureter & 4 & $(10.0)$ & 8 & $(20.0)$ & \\
\hline & Lower ureter & 28 & $(70.0)$ & 20 & $(50.0)$ & \\
\hline Total & & 40 & $(100.0)$ & 40 & $(100.0)$ & \\
\hline
\end{tabular}

is not generally detected until the scheduled stent removal (i.e., the majority of patients did not develop a symptomatic UTI) indicates that the presence of bacterial colonization or even a biofilm on the surface of a stent does not necessarily lead to infection, especially in patients with intact immune systems.

Overall, $66(82.5 \%)$ patients had a postoperative stent and accounted for $8(72.7 \%)$ of the UTIs. So the rate of UTI among postoperative stent patients was $12.1 \%$, thus, indicated that stenting in the present study might not increase the risk of postoperative infection. All of these stents were removed with cystoscopy and antibiotics were used at the time of cystoscopies. Moltzahn et al. reported a 9.5\%UTI rate following ureteroscopy with postoperative stenting and they concluded that continuous low-dose antibiotic treatment during the entire stent-indwelling time did not reduce the quantity or severity of UTIs compared with the peri-interventional antibiotic prophylaxis only [13].

The rate of infection for group 1 was $12.5 \%$, and $15 \%$ for group 2 , but the rate was not significantly different $(\mathrm{p}=0.74)$. Chew et al. reported the rate of UTI in patients with perioperative antibiotics only and patients with continuous post-operative antibiotics (4.8\% and 10.2 , $\mathrm{p}=0.1457$ ) respectively [19]. However, it is important to recognize that failing to reach statistical significance could also be due to small sample size. We do not believe that postoperative antibiotics are associated with increased rates of infection; however, these findings are congruent with the AUA Best Practice Policy Statement, which does not support the use of postoperative antibiotics beyond 24 hours following Ureteroscopy $[8,11]$.

The choice of antibiotic for prophylaxis should take into account the effectiveness, tolerability and the cost. The spectrum must be large enough to cover normal and pathogenic flora. In patients with prolonged hospitalization, we should be aware of the hospital flora and have in mind the risk of potential infection, the target organ and the role of local inflammation [20,21]. For the genitourinary tract, fluoroquinolones, cephalosporins and aminoglycosides have been recommended for antibiotic prophylaxis because of their prolonged half-life, a relatively broad spectrum of action, minimal side effects and low price [22]. Both fluoroquinolones and cephalosporins have proven useful in antibiotic prophylaxis [23] without apparent differences in efficacy between these two antibiotic classes [3].
Although in our hospital there is no standard policy regarding the choice of antibiotic, there are hospitals where antibiotics used for prophylaxis are changed periodically according to the local sensitivity spectrum. Moreover, it should be noted that there are compelling reasons to limit antibiotic prescription to the extent possible. Most notably, the overuse of antibiotics will promote antibiotic resistance, as increasing the exposure of bacterial species to antibiotics unnecessarily will increase their chances of developing resistance mechanisms, making it significantly more difficult to treat them with the antibiotics we currently have available. Infections with MRSA, P. aeruginosa, and Stenotrophomonas are only a few examples of difficult to treat bacterial strains. Exposing bacterial strains to antibiotics unnecessarily will only limit the number of agents we have available to treat the infection.

\section{Conclusions}

In patients undergoing ureteroscopic stone treatment who had a negative baseline urine culture, rates of postoperative UTI was not reduced by continuous postoperative antibiotic prophylaxis and a single dose intraoperative antibiotic is quite sufficient to prevent postoperative UTI. Proper effort should also be made to ensure one is familiar with local antibiograms and accordingly chooses an appropriate preoperative antibiotic. The benefits of implementing this approach across the urological community are likely to include reduced healthcare costs, fewer treatment-related adverse effects, and decreased selective pressure for resistant organisms.

\section{Conflict of interest}

The authors declare that they have no competing interests.

\section{References}

1. Fourcade RO (1990) Antibiotic prophylaxis with cefotaxime in endoscopic extraction of upper urinary tract stones: a randomized study. The Cefotaxime Cooperative Group. J Antimicrob Chemother 26: 77-83.

2. Grabe M (2004) Controversies in antibiotic prophylaxis in urology. Int J Antimicrob Agents 23 Suppl 1: S17-23. [Crossref]

3. Geavlete P, Jecu M, Geavlete B, Multescu R, Nita G, et al. (2010) Ureteroscopy--an essential modern approach in upper urinary tract diagnosis and treatment. $J$ Med Life 3: 193-199. [Crossref]

4. Urban JA (2006) Cost analysis of surgical site infections. Surg Infect (Larchmt) 7 Supp 1: S19-22. [Crossref]

5. Gonen M, Turan H, Ozturk B, Ozkardes H (2008) Factors affecting fever following percutaneous nephrolithotomy: A prospective clinical study. J Endourol 22: 2135-2138.

6. Sharifi Aghdas F, Akhavizadegan H, Aryanpoor A, Inanloo H, Karbakhsh M (2006) Fever after percutaneous nephrolithotomy: Contributing factors. Surg Infect (Larchmt) 7: $367-371$.

7. Chen L, Xu QQ, Li JX, Xiong LL, Wang XF, et al. (2008) Systemic inflammatory response syndrome after percutaneous nephrolithotomy: An assessment of risk factors. Int J Urol 15: 1025-1028.

8. Wolf JS, Bennett CJ, Dmochowski RR, Hollenbeck BK, Pearle MS, et al. (2008) Best practice policy statement on urologic surgery antimicrobial prophylaxis. J Urol 179: 1379-1390.

9. Knopf HJ, Graff HJ, Schulze H (2003) Perioperative antibiotic prophylaxis in ureteroscopic stone removal. Eur Urol 44: 115-118.

10. Ramaswamy K, Shah O (2012) Antibiotic prophylaxis after uncomplicated ureteroscopic stone treatment: Is there a difference? J Endourol 26: 122-125.

11. Preminger GM, Tiselius HG, Assimos DG, Alken P, Buck AC, et al. (2007) 2007 Guideline for the management of ureteral calculi. Eur Urol 52: 1610-1631. [Crossref]

12. Pricop C, Dorobat C, Puia D, Orsolya M (2013) Antibiotic prophylaxis in retrograde ureteroscopy: What strategy should we adopt? Germs 3: 115-121. 
Mama RK (2018) A comparative study between a single dose pre-induction antibiotic versus continuous antibiotics to prevent urinary tract infection during ureteroscopy

13. Moltzahn F, Haeni K, Birkhauser FD, Roth B, Thalmann GN, et al. (2013) Periinterventional antibiotic prophylaxis only vs continuous low-dose antibiotic treatment in patients with JJ stents: A prospective randomized trial analyzing the effect on urinary tract infections and stent-related symptoms. BJU Int 111: 289-295.

14. Ozgur BC, Ekici M, Yuceturk CN, Bayrak O (2013) Bacterial colonization of double J stents and bacteriuria frequency. Kaohsiung J Med Sci 29: 658-661.

15. Cadieux PA, Chew BH, Nott L, Seney S, Elwood CN, et al. (2009) Use of triclosaneluting ureteral stents in patients with long-term stents. J Endourol 23: 1187-1194. [Crossref]

16. Chew BH, Denstedt JD (2004) Technology insight: Novel ureteral stent materials and designs. Nat Clin Pract Urol 1: 44-48. [Crossref]

17. Rahman MA, Alam MM, Shahjamal S, Islam MR, Haque ME (2012) Predictive value of urine cultures in evaluation of bacterial colonization of ureteral stents. Mymensingh Med J 21: 300-305.

18. Tenke P, Köves B, Nagy K, Hultgren SJ, Mendling W, et al. (2012) Update on biofilm infections in the urinary tract. World J Urol 30: 51-57. [Crossref]
19. Chew BH, Flannigan R, Kurtz M, Gershman B, Arsovska O, et al. (2016) A Single Dose of Intraoperative Antibiotics Is Sufficient to Prevent Urinary Tract Infection During Ureteroscopy. J Endourol 30: 63-68. [Crossref]

20. Naber KG, Hofstetter AG, Bruhl P, Bichler KH, Lebert C (2001) Guidelines for the perioperative prophylaxis in urological interventions of the urinary and male genital tract. Int J Antimicrob Agents 17: 321-326.

21. Monga M (2013) Ureteroscopy: Indications, Instrumentation \& Technique. New York: Humana Press.

22. Enzler MJ, Berbari E, Osmon DR (2011) Antimicrobial prophylaxis in adults. Mayo Clin Proc 86: 686-701. [Crossref]

23. Wilson W, Taubert KA, Gewitz M, Lockhart PB, Baddour LM, et al. (2008) Prevention of infective endocarditis: guidelines from the American Heart Association: a guideline from the American Heart Association Rheumatic Fever, Endocarditis and Kawasaki Disease Committee, Council on Cardiovascular Disease in the Young, and the Council on Clinical Cardiology, Council on Cardiovascular Surgery and Anesthesia, and the Quality of Care and Outcomes Research Interdisciplinary Working Group. J Am Dent Assoc 139: 3S-24S.

Copyright: (2018 Mama RK. This is an open-access article distributed under the terms of the Creative Commons Attribution License, which permits unrestricted use, distribution, and reproduction in any medium, provided the original author and source are credited. 